\title{
Fibronectin Mechanobiology Regulates Tumorigenesis
}

\author{
Karin Wang, ${ }^{1,2}$ Bo Ri Seo, ${ }^{2}$ Claudia Fischbach, ${ }^{2,3}$ and Delphine Gourdon ${ }^{1,2}$ \\ ${ }^{1}$ Department of Materials Science and Engineering, Cornell University, 327 Bard Hall, Ithaca, NY 14853, USA; ${ }^{2}$ Department of \\ Biomedical Engineering, Cornell University, Ithaca, NY 14853, USA; and ${ }^{3}$ Kavli Institute at Cornell for Nanoscale Science, \\ Cornell University, Ithaca, NY 14853, USA
}

(Received 6 July 2015; accepted 8 August 2015; published online 15 August 2015)

Associate Editor Michael R. King oversaw the review of this article.

\begin{abstract}
Fibronectin (Fn) is an essential extracellular matrix (ECM) glycoprotein involved in both physiological and pathological processes. The structure-function relationship of Fn has been and is still being studied, as changes in its molecular structure are integral in regulating (or dysregulating) its biological activities via its cell, matrix component, and growth factor binding sites. Fn comprises three types of repeating modules; among them, FnIII modules are mechanically unstable domains that may be extended/unfolded upon cell traction and either uncover cryptic binding sites or disrupt otherwise exposed binding sites. Cells assemble Fn into a fibrillar network; its conformational flexibility implicates Fn as a critical mechanoregulator of the ECM. Fn has been shown to contribute to altered stroma remodeling during tumorigenesis. This review will discuss (i) the significance of the structure-function relationship of Fn at both the molecular and the matrix scales, (ii) the role of Fn mechanobiology in the regulation of tumorigenesis, and (iii) Fn-related advances in cancer therapy development.
\end{abstract}

Keywords-Fibronectin conformational flexibility, Fibronectin mechanics, Tumor stroma, Tumor progression.

\section{Fn AND ITS SIGNIFICANCE IN CANCER}

Fibronectin (Fn) is one of the most abundant extracellular matrix proteins (ECM) along with collagen. Fn was first discovered as a high molecular weight fibroblast cell surface protein in the early 1970 s, ${ }^{64,126}$ and then as an extracellular fibrillar network surrounding fibroblasts through immunofluorescence and scanning electron microscopy. ${ }^{155}$ Early isolation of Fn revealed a dimeric glycoprotein with two subunits measuring $\sim 220 \mathrm{kDa}^{165}$ held together by disulfide

Address correspondence to Delphine Gourdon, Department of Materials Science and Engineering, Cornell University, 327 Bard Hall, Ithaca, NY 14853, USA. Electronic mail: dg434@cornell.edu bonds. ${ }^{66}$ Most Fn is synthesized by hepatocytes to circulate in the bloodstream as soluble plasma Fn. Various cells also secrete Fn, named cellular Fn, to be directly assembled into an insoluble fibrillar network. Plasma and cellular Fn mediate different biological behaviors; plasma Fn is essential in clots during early wound healing, whereas cellular Fn mediates late wound healing, neovascularization, and angiogenesis (Fig. 1a) ${ }^{50,147}$ Fn is also implicated in other physiological (e.g., embryogenesis ${ }^{156}$ ) and pathological ${ }^{30}$ (e.g., fibrosis, cancer) processes.

Originally, Fn was discovered because fibroblast cells lack a cell surface protein after viral transformation. ${ }^{64,155}$ However, the loss of Fn is not a good marker of malignancy, as some anchorage-independent tumorigenic cell lines are still able to assemble a fibrillar Fn network. ${ }^{73}$ Further studies assessing the role of Fn in malignancy reveal high concentrations of plasma Fn after mice were inoculated with Ehrlich tumor cells, ${ }^{167}$ but plasma Fn fluctuates with clinical events such as chemotherapy. ${ }^{24,168}$ Other reports addressed the controversial deposition of Fn in tumors and found that it is absent in tumors but abundant in the surrounding stroma. ${ }^{8,144}$ As such, understanding Fn dynamics, i.e., Fn deposition and remodeling during tumorigenesis, is essential to expanding our knowledge of cancer.

The tumor stroma is a complex microenvironment in which components are recruited or remodeled to facilitate invasive growth and metastasis. ${ }^{43,54}$ Therefore, specific focus is placed on understanding how the surrounding ECM is altered to mediate tumor progression. ${ }^{75,130}$ Cancer-associated fibroblasts (CAFs) are major sources of increased ECM deposition and altered remodeling ${ }^{97}$ to create tracks for cancer cell invasion. ${ }^{46}$ This review will discuss (i) the importance of Fn structure, matrix assembly, and mechanics in invasive tumor growth, and (ii) their relevance to improve therapeutic strategies and diagnostic tools. 


\section{Fn MECHANOREGULATION OF VARIOUS CELLULAR ACTIVITIES}

Fn is a mechanoregulator of the ECM due to its conformational flexibility ${ }^{14,35,38,159}$ in both plasma ${ }^{1}$ and fibrillar forms. ${ }^{2,139}$ Fn consists of 3 repeating modules: FnI, FnII, and FnIII. ${ }^{30,124}$ FnI and FnII are mechanically stable modules as they are stabilized by disulfide bonds, but FnIII lack these disulfide bonds and are sensitive to external mechanical forces. ${ }^{67}$ FnIII modules are made up of $7 \beta$ strands within 2 antiparallel $\beta$ sheets surrounding a hydrophobic core, with FnIII $_{10}$ holding a RGD loop (cell-binding site) between the $\mathrm{F}$ (6th) and $\mathrm{G}$ (7th) $\beta$ strands. ${ }^{96}$ The RGD sequence is a ubiquitous cell binding region as it has also been found in other proteins such as fibrinogen, vitronectin, laminin, and thrombospondin. ${ }^{125}$ Fn contains two sites that collaboratively confer adhesion, ${ }^{112}$ the RGD site on FnIII 10 and the PHSRN synergy site located on the adjacent FnIII $_{9}{ }^{6}$ Simultaneous engagement to both RGD and PHSRN sites is essential for integrins $\alpha_{5} \beta_{1}{ }^{111}$ resulting in a binding that is highly sensitive to Fn molecular conformation. ${ }^{85}$ In contrast, the binding of most other integrins, including $\alpha_{\mathrm{v}} \beta_{3}$ integrins, requires engagement only to the RGD loop and is not (or less) sensitive to Fn conformation. ${ }^{79}$ Briefly, the RGD loop is separated from the PHSRN site by $30-40 \AA$ and a small rotation between FnIII $_{9}$ and FnIII $_{10}$ orients the two cell binding sites on the same side of the Fn molecule. ${ }^{88}$ Therefore, any change either in the orientation (i.e., in the relative angles between the two adjacent modules) or in the spacing between adjacent modules (e.g., as it occurs during FnIII 10 unfolding and shown in Fig. 1b), alters the type of transmembrane receptors used by cells to bind to the Fn matrix, ${ }^{106}$ and the subsequent downstream signaling. Another important region on Fn essential to mechanoregulation is the $\mathrm{FnIII}_{12-14}$ sequence, which binds various growth factors ${ }^{98}$ for sustained, localized signaling. Immobilization of growth factors modulates different downstream signaling. ${ }^{3}$ Specifically, Fn-bound vascular endothelial growth factor (VEGF) mediates structured vascularization whereas soluble VEGF directs large, leaky vasculature. ${ }^{90}$ Thus, Fn conformational flexibility is able to regulate cell activity via integrin specificity and growth factor binding.

Various cells are able to incorporate plasma Fn into the predominantly cellular Fn based-ECM of any tissue $^{32,114}$ Additionally, fibroblasts are able to deposit a Fn matrix by secreting and assembling Fn into fibers at the cell periphery. ${ }^{145}$ Cells' integrins $\alpha_{5} \beta_{1},{ }^{42} \alpha_{3} \beta_{1},{ }^{162}$ and $\alpha_{4} \beta_{1}{ }^{133}$ binding to $\mathrm{Fn}$ were shown to participate in Fn matrix assembly. Assembly requires mechanical stimulation provided by cellular traction forces to induce a conformational change in Fn and expose cryptic binding sites that mediate Fn polymerization. ${ }^{18,92}$ Recent advances in super-resolution microscopy such as direct stochastic optical reconstruction microscopy provide insight to the ordered structure of Fn within bundled fibers, demonstrating that Fn molecules are aligned within fibers with alternating $\mathrm{N}$ terminal and C-terminal overlapping regions. ${ }^{45} \mathrm{Fn}$ maturation follows deposition and involves the polymerization of nascent deoxycholate-soluble Fn (12$20 \mathrm{~nm}$ in diameter) ${ }^{22}$ ultrathin fibrils into mature deoxycholate-insoluble Fn thick fibrils networks (up to $200 \mathrm{~nm}$ in diameter). ${ }^{45,102}$ Although multiple Fn conformations coexist in the matrix (and in individual fibrils), the average Fn conformation has been reported to evolve during ECM maturation from compact/extended Fn in early fibrils to extended/unfolded Fn in mature fibrils and matrices. ${ }^{11,87,139}$ The polymerization of $\mathrm{Fn}$ in extended conformations ${ }^{47}$ stimulates cell growth, ${ }^{142}$ a process that may be mediated by interactions with heparin sulfate proteoglycans (another matrix component to which Fn binds). ${ }^{62,141}$ Fn networks may also be initiated via self-assembly. Fn contains conformational-dependent ${ }^{59}$ binding sites for itself located on $\mathrm{FnI}_{1-5}, \mathrm{FnIII}_{1-2}, \mathrm{FnIII}_{4-5}$, and FnIII $_{12-14}{ }^{45}$ These Fn-Fn interactions may be mediated by interactions with FnIII $_{10} \cdot{ }^{57}$ Furthermore, fragments of these binding sites have been shown to inhibit Fn-Fn interactions and Fn fibrillogenesis. ${ }^{103,108}$ Thus, changes to initial Fn conformations are also crucial in the regulation of Fn binding to other ECM components (including itself), and modulate further ECM deposition and remodeling.

The assembly of an initial Fn network ${ }^{138}$ is often a prerequisite for the downstream deposition of collagen. ${ }^{101,140,148}$ Reciprocally, the co-deposition of collagen has several effects on the initial Fn matrix: it assists further Fn remodeling by matrix metalloproteinases such as MT1-MMP, ${ }^{135}$ it stabilizes the ECM, ${ }^{140}$ it promotes cell proliferation and maintenance of microtissue morphology (ECM reorganization), ${ }^{134}$ and it facilitates cell migration. ${ }^{143}$ The reported co-localization of both Fn and procollagen within the cell further demonstrates a likely synergistic relationship between these two ECM proteins. ${ }^{89}$ Fn contains a large (multimodular) collagen binding site ${ }^{51}$ located on modules $\mathrm{FnI}_{6} \mathrm{FnII}_{1-2} \mathrm{FnI}_{7-9} \cdot{ }^{68} \mathrm{Fn}$ regions within this site $^{119}$ collectively bind ${ }^{76}$ to the collagen $\alpha 1(\mathrm{I})$ chain between residues 757 and $791 .^{33,81}$ Collagen binding stabilizes the $90^{\circ}$ kink between $\mathrm{FnI}_{6} \mathrm{FnII}_{1-2} \mathrm{FnI}_{7}$ and $\mathrm{FnI}_{8-9},{ }^{36}$ which is believed to assist $\mathrm{Fn}$ in maintaining a compact/relaxed conformation in the stroma, further regulating normal tissue homeostasis.

Fn-coated beads restrained by optical traps reveal cells' ability to sense their environment and to respond 


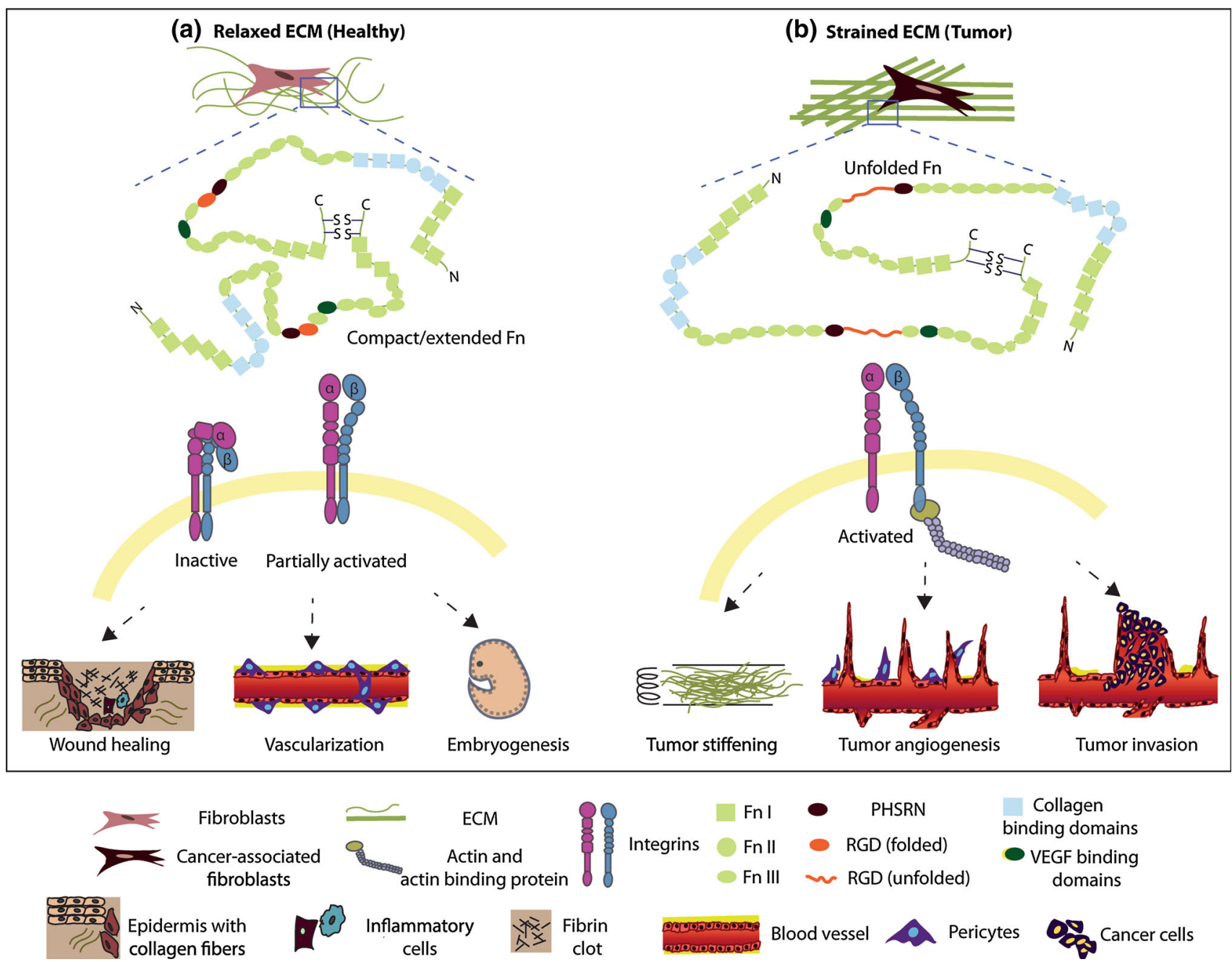

FIGURE 1. Structure-function relationship of fibronectin in healthy and pathological environments. In healthy environments, the ECM is in balance between relaxed and strained conformations to maintain normal tissue homeostasis. However, the ECM in tumor stroma has lost its integrity and starts mediating an altered ECM phenotype. The relaxed ECM (a) contains mostly compact/ extended fibronectin, whereas in the strained ECM (b), fibronectin undergoes conformational changes to resist cell-mediated traction forces. These changes to fibronectin lead to exposure of soluble factor (i.e., VEGF) binding and cryptic sites. Furthermore, these conformational changes facilitate specific matrix component binding to mediate ECM remodeling or modify integrins engagement and activation (i.e., $\alpha v \beta 3$ vs. $\alpha 5 \beta 1$ ) to consequently modulate cell behaviors. Moreover, unfolded Fn is correlated with enhanced fiber strain and bulk ECM stiffening. Therefore, tumor-associated fibronectin assembled in tumor stroma facilitates a cascade of dysregulated downstream signaling for tumor progression.

to increased external resistance ${ }^{25}$ due to the strengthening of cytoskeletal tension, as later confirmed by traction force microcopy. ${ }^{154}$ Additionally, lysophosphatidic acid (from platelets) mediates Rho-activated stress fiber formation and enhances Fn matrix assembly, revealing the importance of cellular tension in Fn fibrillogenesis. ${ }^{12,169,171}$ Briefly, $\alpha_{5} \beta_{1}$ integrins translocating along actin cytoskeletal bundles elongate Fn molecules ${ }^{117}$ with varying amounts of force, ${ }^{113}$ which initiates Fn polymerization and induces cytoskeletal tension. ${ }^{58}$ L8, an antibody known to bind Fn within $\mathrm{FnI}_{9}$ and $\mathrm{FnIII}_{1}$ and to inhibit Fn fiber assembly when added to fibroblast culture medium, ${ }^{23}$ increases its binding to Fn when Fn monolayers deposited on rubber substrates (cell-free system) were mechanically strained to expose a cryptic binding site. ${ }^{173}$ These studies suggest that isolated Fn must unfold to bind to itself and begin the fibrillogenesis process. Detailed analysis of Fn matrix assembly and maturation indicates that Fn fibers are highly elastic ${ }^{65,82,115}$ and heterogeneous as they comprise multiple molecular conformations, from compact/relaxed to extended/ unfolded. ${ }^{87,139}$ The elasticity of Fn fibers can be attributed to the conformational flexibility of FnIII modules (lacking disulfide bonds) that are allowed to extend/unfold upon cellular traction, as suggested by 
steered molecular dynamics simulations ${ }^{86}$ and fluorescence resonance energy transfer. ${ }^{139}$ Importantly, an in vivo study also portrays the critical role of Fn conformational changes in modulating tissue function (e.g., the exposure of $\mathrm{FnIII}_{1}$ mediated by skeletal muscle contraction leads to vasodilation). ${ }^{60}$ Collectively, Fn's cell-induced changes in conformation implicate this glycoprotein as a critical mechanotransducer in translating mechanical signals from the external environment into biochemical signals mediated by integrin clustering and cytoskeletal tension. ${ }^{26,150}$

\section{ROLES OF CONFORMATION AND MECHANICS OF Fn IN TUMORIGENESIS}

In fetal tissues and cancers, cellular Fn is larger ${ }^{100}$ and alternatively spliced ${ }^{132}$ to contain the following sequences: IIICS, ED-A, ED-B, which confer additional conformational changes to Fn. ${ }^{10,16,39,149}$ Fn ED-A is found at sites of tissue remodeling and during dysregulated signaling, it promotes a fibrotic phenotype ${ }^{136}$ for tumorigenesis ${ }^{77}$ and for neovascularization of metastases. ${ }^{127}$ This splice variant enhances VEGF-C secretion via the PI3 K/Akt signaling pathway. ${ }^{163} \mathrm{Fn}$ ED-A secreted by endothelial cells (isolated from tumors) also induces epithelial-mesenchymal transition of cancer cells by activating the FAK-Src signaling pathway via $\alpha_{9} \beta_{1} \cdot{ }^{116,137}$ Instead, Fn ED-B is found in the tumor stroma ${ }^{72}$ and in the tumor vasculature. ${ }^{19}$ This splice variant of Fn has been found to enhance cell adhesion and formation of focal adhesions for cell spreading. ${ }^{56}$ ECM stiffening, a hallmark of cancer, has been found to enhance ED-B splicing of Fn to propagate a tumorigenic phenotype. ${ }^{13}$ Thus, changes in conformation, mechanics, and alternative splicing of Fn synergistically regulate tumorigenesis.

Fn is up-regulated in the tumor stroma. ${ }^{30}$ Its enhanced synthesis ${ }^{110}$ is attributed to CAFs, fibroblasts with altered phenotype and function. ${ }^{74} \mathrm{CAFs}$ are activated by TGF- $\beta^{83}$ or transformed by Fn-tissue transglutaminase complexes contained in microvesicles released from cancer cells. ${ }^{5}$ Breast tumor CAFs deposit an initially dense, unfolded ${ }^{20}$ and stiff ${ }^{153}$ Fn matrix that facilitates an 'integrin switch', i.e., a change from primarily $\alpha_{5} \beta_{1}$ binding (that depends on Fn conformation) to that of mostly $\alpha_{v} \beta_{3}$ binding (that is independent of $\mathrm{Fn}$ conformation), ${ }^{34,151}$ resulting in enhanced pro-angiogenic (VEGF) secretion. ${ }^{152,153}$ Changes to the material properties of Fn can in turn mediate a cascade of signaling events for tumorigenesis (e.g., ECM unfolding, stiffening, tumor angiogenesis, and tumor invasion) (Fig. 1b).
Under conditions of normal tissue homeostasis, Fn mediates strong cellular adhesion. Upon matrix maturation during healthy ECM remodeling (e.g., wound healing, vascularization, embryogenesis) (Fig. 1a), Fn gradually unfolds while cells become more contractile and develop strong fibrillar adhesions containing $\beta_{1}$ integrins. ${ }^{4} \alpha_{5} \beta_{1}$ integrins binding to $\mathrm{Fn}$ stimulates myosin $\mathrm{II}^{44}$ and RhoA-GTPase to form robust peripheral fibrillar adhesions. ${ }^{29}$ These strong adhesive forces between $\mathrm{Fn}$ and $\alpha_{5} \beta_{1}$ integrins $(\sim 93 \mathrm{pN})^{94}$ reduce migration of invasive cells. ${ }^{69}$ Fn conformational changes are often responsible for an 'integrin switch' as Fn conformation alters the type of integrins cells may utilize to bind to the surrounding ECM. As detailed in Section II, the most abundant Fn integrins, $\alpha_{5} \beta_{1}$, require both the synergy and the RGD sites located on $\mathrm{FnIII}_{9}$ and $\mathrm{FnIII}_{10}$, respectively, to form complexes with Fn, which implies that strong $\alpha_{5} \beta_{1}$-Fn binding is conformation-dependent and occurs only when Fn is in a nearly compact conformation. In contrast, $\alpha_{v} \beta_{3}$ integrins require only the RGD site, i.e., weaker $\alpha_{\mathrm{v}} \beta_{3^{-}}$ Fn binding is conformation-independent and occurs even when Fn is unfolded during ECM remodeling. ${ }^{28}$ Weak Fn- $\alpha_{\mathrm{v}} \beta_{3}$ adhesions ${ }^{131}$ by cancer cells then lead to greater cytoskeletal reorganization for enhanced migration capacity ${ }^{9}$ and resistance against anoikis (Fig. 1b). ${ }^{170}$ Once Fn conformation is altered during tumorigenesis, cell-matrix interactions are dysregulated and changes to downstream signaling take place.

As Fn contains binding sites for cells, growth factors, and matrix components, variations in Fn conformation during tumorigenesis alter multiple microenvironmental interactions. The up-regulation of Fn combined with the preferred utilization of $\alpha_{v} \beta_{3}$ in the tumor stroma mediates the release and activation of matrix metalloproteinase-2 (MMP-2), which favors tumor invasion and metastasis. ${ }^{71,128}$ The resulting remodeled Fn, likely degraded by MMP-2, may in turn bind with altered affinity ${ }^{37}$ to collagen ECM, which may lead to the formation of dysregulated, crosslinked, and stiff $\mathrm{Col} \mathrm{I}^{93}$ tracks for enhanced invasion by cancer cells. $^{27,122}$ Although the deposition of collagen usually requires the presence of provisional $\mathrm{Fn}$, enhanced secretion of TGF- $\beta$ does lead to collagen fibrillogenesis and fibrotic ECM remodeling even in the absence of Fn. ${ }^{107}$

Besides conformation, stiffness of the ECM also plays a role in tumorigenesis. ${ }^{55,93,120} \mathrm{ECM}$ stiffening not only promotes Fn ED-B splicing and Fn unfolding for a pro-angiogenic integrin switch, but also contributes to TGF- $\beta$ activity, ${ }^{99}$ a phenomenon that can influence myofibroblast differentiation ${ }^{7,160}$ or epithelial to mesenchymal transition (EMT) for tumor progression. ${ }^{91}$ Invasive cells preferentially migrate towards 
stiffer ECM (durotaxis). ${ }^{95,121}$ Durotaxis is mediated by both the recruitment of $\alpha_{\mathrm{v}} \beta_{3}$ integrins that re-organize and reinforce the cytoskeleton ${ }^{9,123}$ at the leading edge of cells ${ }^{70}$ and the extensions of filopodia. ${ }^{161}$ This rigidity response is attributed to activation of p130Cas via Fyn recruitment by receptor-like protein tyrosine phosphatase alpha (RPTP $\alpha)$ at the leading edge of these cells. ${ }^{84}$ As altered $\mathrm{Fn}$ is stiffer, it may direct cancer cell invasion into the surrounding stroma for eventual metastasis.

Finally, Fn binding to cell surfaces via integrins also mediates clustering of growth factor receptors. ${ }^{164}$ Enhanced levels of $\mathrm{VEGF}^{41}$ are secreted by breast cancer cells (and/or fibroblasts subjected to paracrine signaling by breast cancer cells ${ }^{53}$ ) for tumor angiogenesis. ${ }^{31,48}$ An isoform of VEGF, VEGF 165 , increases breast cancer and endothelial cell migration in presence of Fn (and heparin). ${ }^{104}$ Specifically, Fn forms a complex with VEGF-receptor-2 and $\alpha_{5} \beta_{1}{ }^{78}$ to bind VEGF $^{157}$ on the heparin II binding domain located on FnIII $_{13-14 \cdot}{ }^{158}$ Furthermore, ECM components such as heparin or heparan sulfate facilitate an extended conformation of Fn to enhance VEGF binding ${ }^{105}$ in a $\mathrm{pH}$ dependent manner. ${ }^{49}$ As acidic environments promote tumorigenesis, ${ }^{118}$ low $\mathrm{pH}$ in the tumor stroma may contribute to these changes in Fn conformation and subsequent tumor angiogenesis. Overall, the Fn matrix is not only a mechanotransducing network but also a chemical reservoir of signaling molecules for cells, as Fn-bound VEGF facilitates organized vascular sprouting and branching ${ }^{90}$ via enhanced activation of MAPK through $\beta_{1}$ mediated clustering of VEGFR2. ${ }^{21}$

\section{DEVELOPMENT OF Fn-BASED CANCER THERAPY}

During tumorigenesis, primary structure, conformation, and mechanics of Fn are altered, which clearly affects its multiple biological functions. As the ECM stiffens, alternative splicing of Fn increases, ${ }^{13}$ which leads to additional conformational changes (in an already highly strained and stiff tumor-associated matrix ${ }^{153}$ ) and promotes dysregulated downstream cell-matrix interactions for tumor progression. Targeting this altered Fn during tumorigenesis is therefore extremely desirable. Using phage antibody technology, molecular probes were successfully developed to distinguish between different unfolded (strained) states of Fn. ${ }^{15}$ Additionally, CGS-1 and CGS-2 human antibody fragments were isolated and found to directly target Fn containing ED-B in human tissues as well as in other species. ${ }^{17}$ Another antibody fragment specific for $\mathrm{ED}-\mathrm{B}, \operatorname{scFv}(\mathrm{L} 19)$, was fused to domains of interleukin-12 to enhance cellular immunity, which led to slower tumor growth and reduced metastasis ${ }^{52}$ while injection of a radioactive-homodimer form of the fusion protein, ${ }^{123}$ I-L19 $(\mathrm{scFv})_{2}$, in cancer patients demonstrated the potential to image primary and metastatic tumors noninvasively. ${ }^{129}$ Using E. coli expressing bacterial thioredoxin, therapeutic vaccines specific against ED-A and ED-B were also developed and found to stimulate anti-ED-A and anti-ED-B antibodies to reduce tumor growth. ${ }^{40,63}$ Biologically active fragments of Fn were also utilized, particularly for structure-function experiments portraying the ability of Fn to bind to itself and regulate its own function. ${ }^{61,103}$ Among them, a fragment derived from the first type III repeat in Fn, FnIII ${ }_{1 C}$ (named anastellin), ${ }^{109}$ was reported to inhibit tumor growth, angiogenesis, and metastasis. ${ }^{166}$ In vitro experiments studying the mechanisms behind anastellin's effects revealed that anastellin bound Fn and induced a conformational loss of a Fn epitope containing ED-A, which then activated MAPK and filopodia to stimulate Fn remodeling. ${ }^{80}$ However, anastellin was also shown to mediate an inflammatory and pro-angiogenic phenotype of stromal cells within tumors. ${ }^{172}$ Finally, Fn-derived $\mathrm{N}$-terminal and C-terminal heparin-binding domains, respectively named heparin I and heparin II domains, were also found to inhibit cancer cell adhesion and invasion by reducing $\alpha_{\mathrm{v}} \beta_{3}$ expression and MMP-9 activity. ${ }^{146}$ As advances are made in our knowledge of Fn nanostructure (e.g., splice variants), assembly (e.g., fibrillogenesis, molecular arrangement in fibers, strain-induced conformational changes, remodeling), and mechanics (e.g., contributions of both elastic and viscoelastic properties of Fn to direct cell behavior) during tumorigenesis, the development of therapeutic strategies and diagnostic tools will continue to improve to mitigate tumorigenesis.

\section{CONCLUSIONS AND FUTURE PERSPECTIVES}

Fn is able to trigger a wide range of cellular activities and is extremely dynamic, constantly undergoing remodeling processes where one or more of its essential properties are modified. Modulation of Fn dynamics is likely a strategy for tumor stromal cells to respond to microenvironmental changes (in particular, paracrine signaling from cancer cells) and contributes actively to tumorigenesis. However, because of the reciprocal nature of cell-Fn interactions, it is still unclear whether early $\mathrm{Fn}$ alterations in the tumor microenvironment are a cause or a consequence of the disease.

Numerous Fn-linked tumorigenesis mechanisms still need to be unraveled. As both plasma and cellular Fn play an inextricable role in mediating different biological functions, delineating their respective con- 
tributions during tumorigenesis must be addressed. Neither the mechanisms responsible for Fn assembly into fibers nor the detailed molecular structure of fibers are well understood, which would certainly help in defining the full range of parameters that regulate the Fn structure-function relationship. Although it is now well accepted that Fn assembly is dysregulated during tumorigenesis and leads to altered materials properties of the entire Fn network, it is likely that other microenvironmental disorders, such as altered MMP activity, additionally drive changes in Fn remodeling to predispose the altered ECM for tumor progression. Hence, understanding the means by which early Fn alterations occur during tumorigenesis may pave the way for the development of both diagnostic tools to halt cancer growth at early stages and therapeutics to prevent invasive cancer growth.

Tumor-associated Fn mechanobiology research is critical to deconvolute the diverse materials properties of the dysregulated tumor Fn, i.e., to distinguish among physical (matrix topology, molecular conformation), biochemical (binding affinity, sequestration), and biomechanical (elasticity, viscoelasticity) alterations during disease progression. For example, agingand/or disease-induced Fn conformational changes occurring at the molecular scale (e.g., unfolding) dictate the binding of specific types of growth factors, integrins, and matrix components, which has deep implications in driving tumorigenesis. However, these molecular conformational changes are usually accompanied by concurrent topological and mechanical changes at a larger scale, which makes it difficult to unravel specific mechanisms and their chronology. As such, the recent advances made towards understanding the structure-function relationship of $\mathrm{Fn}$ in tumorigenesis highlight the importance of utilizing interdisciplinary approaches in cancer research.

\section{ACKNOWLEDGMENTS}

Funding by the NSF under award DMR-1352299 (to DG), by the NIH/NCI under award R01 CA185293 (to CF and DG), and by the Cornell Center on the Microenvironment \& Metastasis under award NCI U54 CA143876 (to CF).

\section{CONFLICT OF INTEREST}

The authors Karin Wang, Bo Ri Seo, Claudia Fischbach, and Delphine Gourdon declare no conflict of interest.

\section{ETHICAL STANDARDS}

No human studies were carried out by the authors for this review article. No animal studies were carried out by the authors for this review article.

\section{OPEN ACCESS}

This article is distributed under the terms of the Creative Commons Attribution 4.0 International License (http://creativecommons.org/licenses/by/4.0/), which permits unrestricted use, distribution, and reproduction in any medium, provided you give appropriate credit to the original author(s) and the source, provide a link to the Creative Commons license, and indicate if changes were made.

\section{REFERENCES}

${ }^{1}$ Alexander, S. S., G. Colonna, and H. Edelhoch. The structure and stability of human plasma cold-insoluble globulin. J. Biol. Chem. 254:1501-1505, 1979.

${ }^{2}$ Alexander, S. S., G. Colonna, K. M. Yamada, I. Pastan, and H. Edelhoch. Molecular properties of a major cell surface protein from chick embryo fibroblasts. J. Biol. Chem. 253:5820-5824, 1978.

${ }^{3}$ Anderson, S. M., T. T. Chen, M. L. Iruela-Arispe, and T. Segura. The phosphorylation of vascular endothelial growth factor receptor-2 (VEGFR-2) by engineered surfaces with electrostatically or covalently immobilized VEGF. Biomaterials 30:4618-4628, 2009.

${ }^{4}$ Antia, M., G. Baneyx, K. E. Kubow, and V. Vogel. Fibronectin in aging extracellular matrix fibrils is progressively unfolded by cells and elicits an enhanced rigidity response. Faraday Discuss. 139:229, 2008.

${ }^{5}$ Antonyak, M. A., B. Li, L. K. Boroughs, et al. Cancer cell-derived microvesicles induce transformation by transferring tissue transglutaminase and fibronectin to recipient cells. PNAS 108(12):4852-4857, 2011.

${ }^{6}$ Aota, S., T. Nagai, and K. M. Yamada. Characterization of regions of fibronectin besides the arginine-glycine-aspartic acid sequence required for adhesive function of the cell-binding domain using site-directed mutagenesis. $J$. Biol. Chem. 266:15938-15943, 1991.

${ }^{7}$ Arora, P. D., N. Narani, and C. A. McCulloch. The compliance of collagen gels regulates transforming growth factor-beta induction of alpha-smooth muscle actin in fibroblasts. AJPA 154:871-882, 1999.

${ }^{8}$ Asch, B. B., B. R. Kamat, and N. A. Burstein. Interactions of normal, dysplastic, and malignant mammary epithelial cells with fibronectin in vivo and in vitro. Cancer Res. 41:2115-2125, 1981.

${ }^{9}$ Balcioglu, H. E., H. van Hoorn, D. M. Donato, T. Schmidt, and E. H. J. Danen. The integrin expression profile modulates orientation and dynamics of force transmission at cell-matrix adhesions. J. Cell Biol. 128:1316-1326, 2015.

${ }^{10}$ Balza, E., et al. A novel human fibronectin cryptic sequence unmasked by the insertion of the angiogenesis-associated extra type III domain B. Int. J. Cancer 125:751-758, 2009. 
${ }^{11}$ Baneyx, G., L. Baugh, and V. Vogel. Coexisting conformations of fibronectin in cell culture imaged using fluorescence resonance energy transfer. Proc. Natl. Acad. Sci. U.S.A. 98:14464-14468, 2001.

${ }^{12}$ Baneyx, G., L. Baugh, and V. Vogel. Fibronectin extension and unfolding within cell matrix fibrils controlled by cytoskeletal tension. PNAS 99:5139-5143, 2002.

${ }^{13}$ Bordeleau, F., et al. Tissue stiffness regulates serine/arginine-rich protein-mediated splicing of the extra domain Bfibronectin isoform in tumors. PNAS 112:8314-8319, 2015.

${ }^{14}$ Bradshaw, M. J., and M. L. Smith. Multiscale relationships between fibronectin structure and functional properties. Acta Biomater. 10:1524-1531, 2014.

${ }^{15}$ Cao, L., M. K. Zeller, V. F. Fiore, P. Strane, H. Bermudez, and T. H. Barker. Phage-based molecular probes that discriminate force-induced structural states of fibronectin in vivo. PNAS 109:7251-7256, 2012.

${ }^{16}$ Carnemolla, B., A. Leprini, G. Allemanni, M. Saginati, and L. Zardi. The inclusion of the type III repeat ED-B in the fibronectin molecule generates conformational modifications that unmask a cryptic sequence. J. Biol. Chem. 267:24689-24692, 1992.

${ }^{17}$ Carnemolla, B., et al. Phage antibodies with pan-species recognition of the oncofetal angiogenesis marker fibronectin ED-B domain. Int. J. Cancer 68:397-405, 1996.

${ }^{18}$ Carraher, C. L., and J. E. Schwarzbauer. Regulation of matrix assembly through rigidity-dependent fibronectin conformational changes. J. Biol. Chem. 288:14805-14814, 2013.

${ }^{19}$ Castellani, P., et al. The fibronectin isoform containing the ED-B oncofetal domain: a marker of angiogenesis. Int. J. Cancer 59:612-618, 1994.

${ }^{20}$ Chandler, E. M., M. P. Saunders, C. J. Yoon, D. Gourdon, and C. Fischbach. Adipose progenitor cells increase fibronectin matrix strain and unfolding in breast tumors. Phys. Biol. 8:015008, 2011.

${ }^{21}$ Chen, T. T., A. Luque, S. Lee, S. M. Anderson, T. Segura, and M. L. Iruela-Arispe. Anchorage of VEGF to the extracellular matrix conveys differential signaling responses to endothelial cells. J. Cell Biol. 188:595-609, 2010.

${ }^{22}$ Chen, Y., L. Zardi, and D. M. P. Peters. High-resolution cryo-scanning electron microscopy study of the macromolecular structure of fibronectin fibrils. Scanning 19:349355, 1997.

${ }^{23}$ Chernousov, M. A., A. I. Faerman, M. G. Frid, O. Y. U. Printseva, and V. E. Koteliansky. Monoclonal antibody to fibronectin which inhibits extracellular matrix assembly. FEBS Lett. 217:124-128, 1987.

${ }^{24}$ Choate, J. J., and D. F. Mosher. Fibronectin concentration in plasma of patients with breast cancer, colon cancer, and acute leukemia. Cancer 51:1142-1147, 1983.

${ }^{25}$ Choquet, D., D. P. Felsenfeld, and M. P. Sheetz. Extracellular matrix rigidity causes strengthening of integrincytoskeleton linkages. Cell 88:39-48, 1997.

${ }^{26}$ Cluzel, C., F. Saltel, J. Lussi, F. Paulhe, B. A. Imhof, and B. Wehrle-Haller. The mechanisms and dynamics of (alpha)v(beta) 3 integrin clustering in living cells. J. Cell Biol. 171:383-392, 2005.

${ }^{27}$ Conklin, M. W., et al. Aligned collagen is a prognostic signature for survival in human breast carcinoma. Am. J. Pathol. 178:1221-1232, 2011.

${ }^{28}$ Danen, E. H., S. Aota, A. A. van Kraats, K. M. Yamada, D. J. Ruiter, and G. N. van Muijen. Requirement for the synergy site for cell adhesion to fibronectin depends on the activation state of integrin alpha 5 beta $1 . \mathrm{J}$. Biol. Chem. 270:21612-21618, 1995.

${ }^{29}$ Danen, E. H. J., P. Sonneveld, C. Brakebusch, R. Fässler, and A. Sonnenberg. The fibronectin-binding integrins alpha5beta1 and alphavbeta3 differentially modulate RhoA-GTP loading, organization of cell matrix adhesions, and fibronectin fibrillogenesis. J. Cell Biol. 159:1071-1086, 2002.

${ }^{30}$ D'Ardenne, A. J., and J. O. McGee. Fibronectin in disease. J. Pathol. 142:235-251, 1984.

${ }^{31}$ De, S., O. Razorenova, N. P. McCabe, T. O'Toole, J. Qin, and T. V. Byzova. VEGF-integrin interplay controls tumor growth and vascularization. Proc. Natl. Acad. Sci. U.S.A. 102:7589-7594, 2005.

${ }^{32}$ Deno, D. C., T. M. Saba, and E. P. Lewis. Kinetics of endogenously labeled plasma fibronectin: incorporation into tissues. Am. J. Physiol. 245:R564-R575, 1983.

${ }^{33}$ Dzamba, B. J., H. Wu, R. Jaenisch, and D. M. Peters. Fibronectin binding site in type I collagen regulates fibronectin fibril formation. J. Cell Biol. 121:1165-1172, 1993.

${ }^{34}$ Elosegui-Artola, A., et al. Rigidity sensing and adaptation through regulation of integrin types. Nat. Mater. 13:631637, 2014.

${ }^{35}$ Engel, J., et al. Shapes, domain organizations and flexibility of laminin and fibronectin, two multifunctional proteins of the extracellular matrix. J. Mol. Biol. 150:97-120, 1981

${ }^{36}$ Erat, M. C., B. Sladek, I. D. Campbell, and I. Vakonakis. Structural analysis of collagen type I interactions with human fibronectin reveals a cooperative binding mode. $J$. Biol. Chem. 288(24):17441-17450, 2013.

${ }^{37}$ Erat, M. C., et al. Identification and structural analysis of type I collagen sites in complex with fibronectin fragments. PNAS 106:4195-4200, 2009.

${ }^{38}$ Erickson, H. P., N. Carrell, and J. McDonagh. Fibronectin molecule visualized in electron microscopy: a long, thin, flexible strand. J. Cell Biol. 91:673-678, 1981.

${ }^{39}$ Fattorusso, R., M. Pellecchia, F. Viti, P. Neri, D. Neri, and K. Wüthrich. NMR structure of the human oncofoetal fibronectin ED-B domain, a specific marker for angiogenesis. Structure 7:381-390, 1999.

${ }^{40}$ Femel, J., et al. Therapeutic vaccination against fibronectin ED-A attenuates progression of metastatic breast cancer. Oncotarget 5:12418-12427-10, 2014.

${ }^{41}$ Ferrara, N., H.-P. Gerber, and J. LeCouter. The biology of VEGF and its receptors. Nat. Med. 9:669-676, 2003.

${ }^{42}$ Fogerty, F. J., S. K. Akiyama, K. M. Yamada, and D. F. Mosher. Inhibition of binding of fibronectin to matrix assembly sites by anti-integrin (alpha 5 beta 1 ) antibodies. J. Cell Biol. 111:699-708, 1990.

${ }^{43}$ Friedl, P., and S. Alexander. Cancer invasion and the microenvironment: plasticity and reciprocity. Cell 147:9921009, 2011

${ }^{44}$ Friedland, J. C., M. H. Lee, and D. Boettiger. Mechanically activated integrin switch controls alpha5betal function. Science 323:642-644, 2009.

${ }^{45}$ Früh, S. M., I. Schoen, J. Ries, and V. Vogel. Molecular architecture of native fibronectin fibrils. Nat. Commun. 6:7275, 2015.

${ }^{46}$ Gaggioli, C., et al. Fibroblast-led collective invasion of carcinoma cells with differing roles for RhoGTPases in leading and following cells. Nat. Cell Biol. 9:1392-1400, 2007.

${ }^{47}$ Gildner, C. D., D. C. Roy, C. S. Farrar, and D. C. Hocking. Opposing effects of collagen I and vitronectin on 
fibronectin fibril structure and function. Matrix Biol. 34:33-45, 2014.

${ }^{48}$ Goel, H. L., and A. M. Mercurio. VEGF targets the tumour cell. Nat. Rev. Cancer 13:871-882, 2013.

${ }^{49}$ Goerges, A. L., and M. A. Nugent. pH regulates vascular endothelial growth factor binding to fibronectin: a mechanism for control of extracellular matrix storage and release. J. Biol. Chem. 279:2307-2315, 2004.

${ }^{50}$ Grinnell, F. Fibronectin and wound healing. J. Cell. Biochem. 26:107-116, 1984.

${ }^{51}$ Hahn, L. H., and K. M. Yamada. Identification and isolation of a collagen-binding fragment of the adhesive glycoprotein fibronectin. Proc. Natl. Acad. Sci. U.S.A. 76:1160-1163, 1979.

${ }^{52}$ Halin, C., et al. Enhancement of the antitumor activity of interleukin-12 by targeted delivery to neovasculature. Nat . Biotechnol. 20:264-269, 2002.

${ }^{53}$ Hanahan, D., and L. M. Coussens. Accessories to the crime: functions of cells recruited to the tumor microenvironment. Cancer Cell 21:309-322, 2012.

${ }^{54}$ Hanahan, D., and R. A. Weinberg. The hallmarks of cancer. Cell 100:57-70, 2000.

${ }^{55}$ Hanahan, D., and R. A. Weinberg. Hallmarks of cancer: the next generation. Cell 144:646-674, 2011.

${ }^{56}$ Hashimoto-Uoshima, M., Y. Z. Yan, G. Schneider, and I. Aukhil. The alternatively spliced domains EIIIB and EIIIA of human fibronectin affect cell adhesion and spreading. $J$. Cell Biol. 110(Pt 18):2271-2280, 1997.

${ }^{57}$ Hocking, D. C., R. K. Smith, and P. J. McKeown-Longo. A novel role for the integrin-binding III-10 module in fibronectin matrix assembly. J. Cell Biol. 133:431-444, 1996.

${ }^{58}$ Hocking, D. C., J. Sottile, and K. J. Langenbach. Stimulation of integrin-mediated cell contractility by fibronectin polymerization. J. Biol. Chem. 275:10673-10682, 2000.

${ }^{59}$ Hocking, D. C., J. Sottile, and P. J. McKeown-Longo. Fibronectin's III-1 module contains a conformation-dependent binding site for the amino-terminal region of fibronectin. J. Biol. Chem. 269:19183-19187, 1994.

${ }^{60}$ Hocking, D. C., P. A. Titus, R. Sumagin, and I. H. Sarelius. Extracellular matrix fibronectin mechanically couples skeletal muscle contraction with local vasodilation. Circ. Res. 102:372-379, 2008.

${ }^{61}$ Homandberg, G. A., J. Kramer-Bjerke, D. Grant, G. Christianson, and R. Eisenstein. Heparin-binding fragments of fibronectin are potent inhibitors of endothelial cell growth: structure-function correlations. Biochim. Biophys. Acta 874:327-332, 1986.

${ }^{62}$ Hubbard, B., J. A. Buczek-Thomas, M. A. Nugent, and M. L. Smith. Heparin-dependent regulation of fibronectin matrix conformation. Matrix Biol. 34:124-131, 2013.

${ }^{63}$ Huijbers, E. J. M., et al. Vaccination against the extra domain-B of fibronectin as a novel tumor therapy. FASEB J. 24:4535-4544, 2010.

${ }^{64}$ Hynes, R. O. Alteration of cell-surface proteins by viral transformation and by proteolysis. Proc. Natl. Acad. Sci. USA 70:3170-3174, 1973.

${ }^{65}$ Hynes, R. O. The dynamic dialogue between cells and matrices: implications of fibronectin's elasticity. Proc. Natl. Acad. USA 96(6):2588-2590, 1999.

${ }^{66}$ Hynes, R. O., and A. Destree. Extensive disulfide bonding at the mammalian cell surface. Proc. Natl. Acad. Sci. USA 74:2855-2859, 1977

${ }^{67}$ Hynes, R. O., and K. M. Yamada. Fibronectins: multifunctional modular glycoproteins. J. Cell Biol. 95:369-377, 1982.
${ }^{68}$ Ingham, K. C., S. A. Brew, and M. M. Migliorini. Further localization of the gelatin-binding determinants within fibronectin. Active fragments devoid of type II homologous repeat modules. J. Biol. Chem. 264:16977-16980, 1989.

${ }^{69}$ Jia, D., I. Entersz, C. Butler, and R. A. Foty. Fibronectin matrix-mediated cohesion suppresses invasion of prostate cancer cells. BMC Cancer 12:94, 2012.

${ }^{70}$ Jiang, G., A. H. Huang, Y. Cai, M. Tanase, and M. P. Sheetz. Rigidity sensing at the leading edge through alphavbeta3 integrins and RPTPalpha. Biophys. J. 90:1804-1809, 2006.

${ }^{71}$ Jiao, Y., et al. Matrix metalloproteinase-2 promotes $\alpha \mathrm{v} \beta 3$ integrin-mediated adhesion and migration of human melanoma cells by cleaving fibronectin. PLoS One 7:e41591, 2012.

${ }^{72}$ Kaczmarek, J., P. Castellani, G. Nicolo, B. Spina, G. Allemanni, and L. Zardi. Distribution of oncofetal fibronectin isoforms in normal, hyperplastic and neoplastic human breast tissues. Int. J. Cancer 59:11-16, 1994.

${ }^{73}$ Kahn, P., and S. I. Shin. Cellular tumorigenicity in nude mice. Test of associations among loss of cell-surface fibronectin, anchorage independence, and tumor-forming ability. J. Cell Biol. 82:1-16, 1979.

${ }^{74}$ Kalluri, R., and M. Zeisberg. Fibroblasts in cancer. Nat. Rev. Cancer 6:392-401, 2006.

${ }^{75}$ Kass, L., J. T. Erler, M. Dembo, and V. M. Weaver. Mammary epithelial cell: influence of extracellular matrix composition and organization during development and tumorigenesis. Int. J. Biochem. Cell Biol. 39:1987-1994, 2007.

${ }^{76}$ Katagiri, Y., S. A. Brew, and K. C. Ingham. All six modules of the gelatin-binding domain of fibronectin are required for full affinity. J. Biol. Chem. 278:11897-11902, 2003.

${ }^{77}$ Kelsh, R. M., P. J. McKeown-Longo, and R. A. F. Clark. EDA fibronectin in keloids create a vicious cycle of fibrotic tumor formation. J. Investig. Dermatol. 135:1714-1718, 2015.

${ }^{78}$ Kim, S., K. Bell, S. A. Mousa, and J. A. Varner. Regulation of angiogenesis in vivo by ligation of integrin alpha5betal with the central cell-binding domain of fibronectin. AJPA 156:1345-1362, 2000.

${ }^{79}$ Kimizuka, F., et al. Role of type III homology repeats in cell adhesive function within the cell-binding domain of fibronectin. J. Biol. Chem. 266:3045-3051, 1991.

${ }^{80}$ Klein, R. M., M. Zheng, A. Ambesi, L. Van De Water, and P. J. McKeown-Longo. Stimulation of extracellular matrix remodeling by the first type III repeat in fibronectin. $J$. Cell Biol. 116:4663-4674, 2003.

${ }^{81}$ Kleinman, H. K., E. B. McGoodwin, G. R. Martin, R. J. Klebe, P. P. Fietzek, and D. E. Woolley. Localization of the binding site for cell attachment in the alpha1(I) chain of collagen. J. Biol. Chem. 253:5642-5646, 1978.

${ }^{82}$ Klotzsch, E., et al. Fibronectin forms the most extensible biological fibers displaying switchable force-exposed cryptic binding sites. Proc. Natl. Acad. Sci. USA 106:18267$18272,2009$.

${ }^{83}$ Kojima, Y., et al. Autocrine TGF-beta and stromal cell-derived factor-1 (SDF-1) signaling drives the evolution of tumor-promoting mammary stromal myofibroblasts. PNAS 107:20009-20014, 2010.

${ }^{84}$ Kostic, A., and M. P. Sheetz. Fibronectin rigidity response through Fyn and p130Cas recruitment to the leading edge. Mol. Biol. Cell 17:2684-2695, 2006. 
${ }^{85}$ Krammer, A., D. Craig, W. E. Thomas, K. Schulten, and V. Vogel. A structural model for force regulated integrin binding to fibronectin's RGD-synergy site. Matrix Biol. 21(2):139-147, 2002.

${ }^{86}$ Krammer, A., H. Lu, B. Isralewitz, K. Schulten, and V. Vogel. Forced unfolding of the fibronectin type III module reveals a tensile molecular recognition switch. PNAS 96:1351-1356, 1999.

${ }^{87}$ Kubow, K. E., E. Klotzsch, M. L. Smith, D. Gourdon, W. C. Little, and V. Vogel. Crosslinking of cell-derived 3D scaffolds up-regulates the stretching and unfolding of new extracellular matrix assembled by reseeded cells. Integr. Biol. 1:635-648, 2009.

${ }^{88}$ Leahy, D. J., I. Aukhil, and H. P. Erickson. 2.0 A crystal structure of a four-domain segment of human fibronectin encompassing the RGD loop and synergy region. Cell 84:155-164, 1996.

${ }^{89}$ Ledger, P. W., N. Uchida, and M. L. Tanzer. Immunocytochemical localization of procollagen and fibronectin in human fibroblasts: effects of the monovalent ionophore, monensin. J. Cell Biol. 87:663-671, 1980.

${ }^{90}$ Lee, S., S. M. Jilani, G. V. Nikolova, D. Carpizo, and M. L. Iruela-Arispe. Processing of VEGF-A by matrix metalloproteinases regulates bioavailability and vascular patterning in tumors. J. Cell Biol. 169:681-691, 2005.

${ }^{91}$ Leight, J. L., M. A. Wozniak, S. Chen, M. L. Lynch, and C. S. Chen. Matrix rigidity regulates a switch between TGF-1-induced apoptosis and epithelial-mesenchymal transition. Mol. Biol. Cell 23:781-791, 2012.

${ }^{92}$ Lemmon, C. A., C. S. Chen, and L. H. Romer. Cell traction forces direct fibronectin matrix assembly. Biophys. J. 96:729-738, 2009.

${ }^{93}$ Levental, K. R., et al. Matrix crosslinking forces tumor progression by enhancing integrin signaling. Cell 139:891906, 2009.

${ }^{94}$ Li, F., S. D. Redick, H. P. Erickson, and V. T. Moy. Force measurements of the a $5 \mathrm{~b} 1$ integrin-fibronectin interaction. Biophys. J. 84:1252-1262, 2003.

${ }^{95}$ Lo, C. M., H. B. Wang, M. Dembo, and Y. L. Wang. Cell movement is guided by the rigidity of the substrate. Biophys. J. 79:144-152, 2000.

${ }^{96}$ Main, A. L., T. S. Harvey, M. Baron, J. Boyd, and I. D. Campbell. The three-dimensional structure of the tenth type III module of fibronectin: an insight into RGD-mediated interactions. Cell 71:671-678, 1992.

${ }^{97}$ Malik, R., P. I. Lelkes, and E. Cukierman. Biomechanical and biochemical remodeling of stromal extracellular matrix in cancer. Trends Biotechnol. 33(4):230-236, 2015.

${ }^{98}$ Martino, M. M., and J. A. Hubbell. The $12^{\text {th }}-14$ th type III repeats of fibronectin function as a highly promiscuous growth factor-binding domain. FASEB J. 24:4711-4721, 2010.

${ }^{99}$ Massague, J. TGFbeta in cancer. Cell 134:215-230, 2008.

${ }^{100}$ Matsuura, H., and S. Hakomori. The oncofetal domain of fibronectin defined by monoclonal antibody FDC-6: its presence in fibronectins from fetal and tumor tissues and its absence in those from normal adult tissues and plasma. Proc. Natl. Acad. Sci. USA 82:6517-6521, 1985.

${ }^{101}$ McDonald, J. A., D. G. Kelley, and T. J. Broekelmann. Role of fibronectin in collagen deposition: Fab' to the gelatin-binding domain of fibronectin inhibits both fibronectin and collagen organization in fibroblast extracellular matrix. J. Cell Biol. 92:485-492, 1982.
${ }^{102}$ McKeown-Longo, P. J., and D. F. Mosher. Binding of plasma fibronectin to cell layers of human skin fibroblasts. J. Cell Biol. 97:466-472, 1983.

${ }^{103}$ McKeown-Longo, P. J., and D. F. Mosher. Interaction of the 70,000-mol-wt amino-terminal fragment of fibronectin with the matrix-assembly receptor of fibroblasts. J. Cell Biol. 100:364-374, 1985.

${ }^{104}$ Miralem, T., R. Steinberg, D. Price, and H. Avraham. VEGF165 requires extracellular matrix components to induce mitogenic effects and migratory response in breast cancer cells. Oncogene 20:5511-5524, 2001.

${ }^{105}$ Mitsi, M., Z. Hong, C. E. Costello, and M. A. Nugent. Heparin-mediated conformational changes in fibronectin expose vascular endothelial growth factor binding sites. Biochemistry 45(34):10319-10328, 2006.

${ }^{106}$ Miyamoto, S., B. Z. Katz, R. M. Lafrenie, and K. M. Yamada. Fibronectin and integrins in cell adhesion, signaling, and morphogenesis. Morphog. Cell. Interact. 857:119-129, 1998.

${ }^{107}$ Moriya, K., et al. A fibronectin-independent mechanism of collagen fibrillogenesis in adult liver remodeling. Gastroenterology 140:1653-1663, 2011.

${ }^{108}$ Morla, A., and E. Ruoslahti. A fibronectin self-assembly site involved in fibronectin matrix assembly: reconstruction in a synthetic peptide. J. Cell Biol. 118:421-429, 1992.

${ }^{109}$ Morla, A., Z. Zhang, and E. Ruoslahti. Superfibronectin is a functionally distinct form of fibronectin. Nature 367:193-196, 1994

${ }^{110}$ Moro, L., M. Colombi, M. P. Molinari Tosatti, and S. Barlati. Study of fibronectin and mRNA in human laryngeal and ectocervical carcinomas by in situ hybridization and image analysis. Int. J. Cancer 51:692697, 1992.

${ }^{111}$ Nagai, T., et al. Monoclonal antibody characterization of two distant sites required for function of the central cellbinding domain of fibronectin in cell adhesion, cell migration, and matrix assembly. J. Cell Biol. 114:12951305, 1991.

${ }^{112}$ Obara, M., M. S. Kang, and K. M. Yamada. Site-directed mutagenesis of the cell-binding domain of human fibronectin: separable, synergistic sites mediate adhesive function. Cell 53:649-657, 1988.

${ }^{113}$ Oberhauser, A. F., C. Badilla-Fernandez, M. CarrionVazquez, and J. M. Fernandez. The mechanical hierarchies of fibronectin observed with single-molecule AFM. J. Mol. Biol. 319:433-447, 2002.

${ }^{114}$ Oh, E., M. Pierschbacher, and E. Ruoslahti. Deposition of plasma fibronectin in tissues. Proc. Natl. Acad. Sci. USA 78:3218-3221, 1981.

${ }^{115}$ Ohashi, T., D. P. Kiehart, and H. P. Erickson. Dynamics and elasticity of the fibronectin matrix in living cell culture visualized by fibronectin-green fluorescent protein. PNAS 96:2153-2158, 1999.

${ }^{116} \mathrm{Ou}$, J., et al. Endothelial cell-derived fibronectin extra domain A promotes colorectal cancer metastasis via inducing epithelial-mesenchymal transition. Carcinogenesis 35:1661-1670, 2014.

${ }^{117}$ Pankov, R., et al. Integrin dynamics and matrix assembly: tensin-dependent translocation of alpha(5)beta(1) integrins promotes early fibronectin fibrillogenesis. J. Cell Biol. 148:1075-1090, 2000.

${ }^{118}$ Peppicelli, S., F. Bianchini, and L. Calorini. Extracellular acidity, a "reappreciated" trait of tumor environment 
driving malignancy: perspectives in diagnosis and therapy. Cancer Metastasis Rev. 33:823-832, 2014.

${ }^{119}$ Pickford, A. R., S. P. Smith, D. Staunton, J. Boyd, and I. D. Campbell. The hairpin structure of the (6)F1(1)F2(2)F2 fragment from human fibronectin enhances gelatin binding. EMBO J. 20:1519-1529, 2001.

${ }^{120}$ Pickup, M. W., J. K. Mouw, and V. M. Weaver. The extracellular matrix modulates the hallmarks of cancer. EMBO Rep. 15:1243-1253, 2014.

${ }^{121}$ Plotnikov, S. V., A. M. Pasapera, B. Sabass, and C. M. Waterman. Force fluctuations within focal adhesions mediate ECM-rigidity sensing to guide directed cell migration. Cell 151:1513-1527, 2012.

${ }^{122}$ Provenzano, P. P., K. W. Eliceiri, J. M. Campbell, D. R. Inman, J. G. White, and P. J. Keely. Collagen reorganization at the tumor-stromal interface facilitates local invasion. BMC Med. 4:38, 2006.

${ }^{123}$ Roca-Cusachs, P., N. C. Gauthier, A. del Rio, and M. P. Sheetz. Clustering of alpha(5)beta(1) integrins determines adhesion strength whereas alpha(v)beta(3) and talin enable mechanotransduction. PNAS 106:16245-16250, 2009.

${ }^{124}$ Ruoslahti, E. Fibronectin and its receptors. Annu. Rev. Biochem. 57(1):375-413, 1988.

${ }^{125}$ Ruoslahti, E. RGD and other recognition sequences for integrins. Annu. Rev. Cell Dev. Biol. 12:697-715, 1996.

${ }^{126}$ Ruoslahti, E., A. Vaheri, P. Kuusela, and E. Linder. Fibroblast surface antigen: a new serum protein. Biochim. Biophys. Acta 322(2):352-358, 1973.

${ }^{127}$ Rybak, J. N., C. Roesli, M. Kaspar, A. Villa, and D. Neri. The extra-domain a of fibronectin is a vascular marker of solid tumors and metastases. Cancer Res. 67:10948-10957, 2007.

${ }^{128}$ Saad, S., D. J. Gottlieb, K. F. Bradstock, C. M. Overall, and L. J. Bendall. Cancer cell-associated fibronectin induces release of matrix metalloproteinase- 2 from normal fibroblasts. Cancer Res. 62:283-289, 2002.

${ }^{129}$ Santimaria, M., et al. Immunoscintigraphic detection of the ED-B domain of fibronectin, a marker of angiogenesis, in patients with cancer. Clin. Cancer Res. 9:571-579, 2003.

${ }^{130}$ Schedin, P., and P. J. Keely. Mammary Gland ECM remodeling, stiffness, and mechanosignaling in normal development and tumor progression. Cold Spring Harbor Perspect. Biol. 3:a003228-a003228, 2011.

${ }^{131}$ Schiller, H. B., et al. $\beta 1-$ and $\alpha$ v-class integrins cooperate to regulate myosin II during rigidity sensing of fibronectinbased microenvironments. Nat. Cell Biol. 15:625-636, 2013.

${ }^{132}$ Schwarzbauer, J. E., J. I. Paul, and R. O. Hynes. On the origin of species of fibronectin. Proc. Natl. Acad. Sci. USA 82:1424-1428, 1985.

${ }^{133}$ Sechler, J. L., A. M. Cumiskey, D. M. Gazzola, and J. E. Schwarzbauer. A novel RGD-independent fibronectin assembly pathway initiated by alpha4betal integrin binding to the alternatively spliced $\mathrm{V}$ region. J. Cell Biol. 113(Pt 8):1491-1498, 2000.

${ }^{134}$ Sevilla, C. A., D. Dalecki, and D. C. Hocking. Regional fibronectin and collagen fibril co-assembly directs cell proliferation and microtissue morphology. PLoS One 8:e77316, 2013.

${ }^{135}$ Shi, F., and J. Sottile. MT1-MMP regulates the turnover and endocytosis of extracellular matrix fibronectin. J. Cell Biol. 124:4039-4050, 2011.

${ }^{136}$ Shinde, A. V., R. Kelsh, J. H. Peters, K. Sekiguchi, L. Van De Water, and P. J. McKeown-Longo. The $\alpha 4 \beta 1$ integrin and the EDA domain of fibronectin regulate a profibrotic phenotype in dermal fibroblasts. Matrix Biol. 41:26-35, 2014.

${ }^{137}$ Shinde, A. V., et al. Identification of the peptide sequences within the EIIIA (EDA) segment of fibronectin that mediate integrin alpha9beta1-dependent cellular activities. J. Biol. Chem. 283:2858-2870, 2008.

${ }^{138}$ Singh, P., C. Carraher, and J. E. Schwarzbauer. Assembly of fibronectin extracellular matrix. Annu. Rev. Cell Dev. Biol. 26:397-419, 2010.

${ }^{139}$ Smith, M. L., et al. Force-induced unfolding of fibronectin in the extracellular matrix of living cells. PLoS Biol. 5:e268, 2007.

${ }^{140}$ Sottile, J., and D. C. Hocking. Fibronectin polymerization regulates the composition and stability of extracellular matrix fibrils and cell-matrix adhesions. Mol. Biol. Cell 13:3546-3559, 2002.

${ }^{141}$ Sottile, J., D. C. Hocking, and K. J. Langenbach. Fibronectin polymerization stimulates cell growth by RGDdependent and -independent mechanisms. J. Cell Biol. 113(Pt 23):4287-4299, 2000.

${ }^{142}$ Sottile, J., D. C. Hocking, and P. J. Swiatek. Fibronectin matrix assembly enhances adhesion-dependent cell growth. J. Cell Biol. 111(Pt 19):2933-2943, 1998.

${ }^{143}$ Sottile, J., F. Shi, I. Rublyevska, H. Y. Chiang, J. Lust, and J. Chandler. Fibronectin-dependent collagen I deposition modulates the cell response to fibronectin. AJP 293:C1934-C1946, 2007.

${ }^{144}$ Stenman, S., and A. Vaheri. Fibronectin in human solid tumors. Int. J. Cancer 27:427-435, 1981.

${ }^{145}$ Steward, R. L., C.-M. Cheng, J. D. Ye, R. M. Bellin, and P. R. LeDuc. Mechanical stretch and shear flow induced reorganization and recruitment of fibronectin in fibroblasts. Sci. Rep. 1:147, 2011.

${ }^{146}$ Tang, N.-H., et al. N-terminal and C-terminal heparinbinding domain polypeptides derived from fibronectin reduce adhesion and invasion of liver cancer cells. $B M C$ Cancer 10:552, 2010.

${ }^{147}$ To, W. S., and K. S. Midwood. Plasma and cellular fibronectin: distinct and independent functions during tissue repair. Fibrogen. Tissue Repair 4:21, 2011.

${ }^{148}$ Velling, T., J. Risteli, K. Wennerberg, D. F. Mosher, and S. Johansson. Polymerization of type I and III collagens is dependent on fibronectin and enhanced by integrins alpha 11 beta 1 and alpha 2beta 1. J. Biol. Chem. 277:3737737381, 2002.

${ }^{149}$ Ventura, E., et al. Alternative splicing of the angiogenesis associated extra-domain $\mathrm{B}$ of fibronectin regulates the accessibility of the B-C loop of the type III repeat 8. PLoS One 5:e9145, 2010

${ }^{150}$ Vogel, V., W. E. Thomas, D. W. Craig, A. Krammer, and G. Baneyx. Structural insights into the mechanical regulation of molecular recognition sites. Trends Biotechnol. 19:416-423, 2001.

${ }^{151}$ Wan, A. M. D., R. M. Schur, C. K. Ober, C. Fischbach, D. Gourdon, and G. G. Malliaras. Electrical control of protein conformation. Adv. Mater. 24:2501-2505, 2012.

${ }^{152}$ Wan, A. M. D., et al. Fibronectin conformation regulates the proangiogenic capability of tumor-associated adipogenic stromal cells. Acta: Biochim. Biophys, 2013.

${ }^{153}$ Wang, K., R. C. Andresen Eguiluz, F. Wu, B. R. Seo, C. Fischbach, and D. Gourdon. Stiffening and unfolding of early deposited-fibronectin increase proangiogenic factor secretion by breast cancer-associated stromal cells. Biomaterials 54:63-71, 2015 
${ }^{154}$ Wang, N., et al. Mechanical behavior in living cells consistent with the tensegrity model. Proc. Natl. Acad. Sci. USA 98:7765-7770, 2001.

${ }^{155}$ Wartiovaara, J. Distribution of fibroblast surface antigen: association with fibrillar structures of normal cells and loss upon viral transformation. J. Exp. Med. 140:1522-1533, 1974.

${ }^{156}$ Wartiovaara, J., I. Leivo, and A. Vaheri. Expression of the cell surface-associated glycoprotein, fibronectin, in the early mouse embryo. Dev. Biol. 69:247-257, 1979.

${ }^{157}$ Wijelath, E. S., et al. Novel vascular endothelial growth factor binding domains of fibronectin enhance vascular endothelial growth factor biological activity. Circ. Res. 91:25-31, 2002.

${ }^{158}$ Wijelath, E. S., et al. Heparin-II domain of fibronectin is a vascular endothelial growth factor-binding domain: enhancement of VEGF biological activity by a singular growth factor/matrix protein synergism. Circ. Res. 99:853-860, 2006.

${ }^{159}$ Williams, E. C., P. A. Janmey, J. D. Ferry, and D. F. Mosher. Conformational states of fibronectin. Effects of $\mathrm{pH}$, ionic strength, and collagen binding. J. Biol. Chem. 257:14973-14978, 1982.

${ }^{160}$ Wipff, P.-J., D. B. Rifkin, J.-J. Meister, and B. Hinz. Myofibroblast contraction activates latent TGF-beta1 from the extracellular matrix. J. Cell Biol. 179:1311-1323, 2007.

${ }^{161}$ Wong, S., W.-H. Guo, and Y.-L. Wang. Fibroblasts probe substrate rigidity with filopodia extensions before occupying an area. PNAS 111:17176-17181, 2014.

${ }^{162} \mathrm{Wu}, \mathrm{C}$., A. E. Chung, and J. A. McDonald. A novel role for alpha 3 beta 1 integrins in extracellular matrix assembly. J. Cell Biol. 108(Pt 6):2511-2523, 1995.

${ }^{163}$ Xiang, L., G. Xie, J. Ou, X. Wei, F. Pan, and H. Liang. The extra domain A of fibronectin increases VEGF-C expression in colorectal carcinoma involving the PI3 K/ AKT signaling pathway. PLoS One 7:e35378, 2012.

${ }^{164}$ Yamada, K. M., and S. Even-Ram. Integrin regulation of growth factor receptors. Nat. Cell Biol. 4(4):E75-E76, 2002.
${ }^{165}$ Yamada, K. M., and J. A. Weston. Isolation of a major cell surface glycoprotein from fibroblasts. Proc. Natl. Acad. Sci. USA 71:3492-3496, 1974.

${ }^{166} \mathrm{Yi}, \mathrm{M}$., and E. Ruoslahti. A fibronectin fragment inhibits tumor growth, angiogenesis, and metastasis. Proc. Natl. Acad. Sci. USA 98:620-624, 2001.

${ }^{167}$ Zardi, L., C. Cecconi, O. Barbieri, B. Carnemolla, M. Picca, and L. Santi. Concentration of fibronectin in plasma of tumor-bearing mice and synthesis by Ehrlich ascites tumor cells. Cancer Res. 39:3774-3779, 1979.

${ }^{168}$ Zerlauth, G., and G. Wolf. Plasma fibronectin as a marker for cancer and other diseases. Am. J. Med. 77:685-689, 1984.

${ }^{169}$ Zhang, Q., W. J. Checovich, D. M. Peters, R. M. Albrecht, and D. F. Mosher. Modulation of cell surface fibronectin assembly sites by lysophosphatidic acid. J. Cell Biol. 127:1447-1459, 1994.

${ }^{170}$ Zhang, Y., H. Lu, P. Dazin, and Y. Kapila. Squamous cell carcinoma cell aggregates escape suspension-induced, p53mediated anoikis: fibronectin and integrin alphav mediate survival signals through focal adhesion kinase. J. Biol. Chem. 279:48342-48349, 2004.

${ }^{171}$ Zhang, Q., M. K. Magnusson, and D. F. Mosher. Lysophosphatidic acid and microtubule-destabilizing agents stimulate fibronectin matrix assembly through Rho-dependent actin stress fiber formation and cell contraction. Mol. Biol. Cell 8:1415-1425, 1997.

${ }^{172}$ Zheng, M., D. M. Jones, C. Horzempa, A. Prasad, and P. J. McKeown-Longo. The first type III domain of fibronectin is associated with the expression of cytokines within the lung tumor microenvironment. J. Cancer 2:478483, 2011.

${ }^{173}$ Zhong, C., M. Chrzanowska-Wodnicka, J. Brown, A. Shaub, A. M. Belkin, and K. Burridge. Rho-mediated contractility exposes a cryptic site in fibronectin and induces fibronectin matrix assembly. J. Cell Biol. 141:539$551,1998$. 